\title{
Part one: conclusion
}

The three elements of sound give us an extremely large palette for sonic branding and technology gives us an almost limitless number of locations and media where the palette can be employed by a brand. Given the diversity of the opportunity, it is unsurprising that most brands are yet to approach their use of sound from a consistent brand perspective. The choices available are vast and the experience, information and research required to make the right choices are highly specialized. As a result, the vast majority of brands have a hotchpotch of sounds representing them. Approach almost any brand and you will discover that the sound for each touchpoint is completely different. Some of it is good and 'on-brand', some of it is bad and 'off-brand'. Our clients most often ask us how we approach the difficult issue of creating consistency across the many media channels and physical touchpoints of a brand.

The answer is simple. There may be 100 different media channels but people have only one set of ears. We think about the human beings that will hear our work rather than the medium and go from there. The essence of sonic branding is to generate consistency across touchpoints and give brands the creative and strategic knowledge to understand what their sonic branding does for their brand. We are all just waking up to the opportunity and finding out about the range of opportunities in the age of sonic touchpoints. In doing so, we are discovering new ways to reward stakeholders. Ultimately, we are building trust and belief through a fundamental emotional communicator that is the match for any other expression of a brand from a graphic logo to a scent.

Sound relates to almost every discipline in branding and has a role in the creation as well as the communication of brands. Sonicbrand uses music to help clients understand what their brands stand for before we help them define how their brands should sound. We can do this because sound and music in particular are universal languages of emotion.

The 'Intel in Capri' story started our journey but the relationship between sound and brands is much more interesting than that. Sound, as we have shown, is exciting and diverse. Branding is just as broad and needs to be understood before we can really evaluate the role of sound in context. In order to progress, then, we have to examine the true nature of brands, how they are created and where sonic fits into their world. 